\title{
Low-Grade Vulvar and Vaginal Intraepithelial Neoplasia: Correlation of Histologic Features with Human Papillomavirus DNA Detection and MIB-1 Immunostaining
}

Sanjay Logani, M.D., Danielle Lu, M.D., Wim G. V. Quint, Ph.D., Lora H. Ellenson, M.D., Edyta C. Pirog, M.D.

Department of Pathology, Emory University School of Medicine, Atlanta, GA (SL); Department of Pathology, Washington University School of Medicine, St. Louis, MO (DL); Academic Medical Center, Amsterdam, The Netherlands (WGVQ); and Department of Pathology, Weill Medical College of Cornell University, NY, NY (LHE, ECP)

Histologic criteria of low-grade vulvar/vaginal intraepithelial neoplasia (VIN1/VAIN1) are well established; however, a significant interobserver variability in diagnosing VIN1/VAIN1 has been reported. The goal of this study was to evaluate the utility of MIB-1 immunostaining as an adjunct test to increase the diagnostic accuracy in equivocal cases of VIN1/VAIN1. The second goal was to examine the distribution of low- and high-oncogenic risk human papillomaviruses (HPVs) in VIN1/VAIN1 lesions. Consecutive vulvar/vaginal biopsies originally diagnosed as VIN1/VAIN1 $(n=43)$ or benign $(n=20)$ were reviewed by two pathologists to obtain a consensus diagnosis. The diagnosis was further confirmed with HPV testing using Short PCR Fragment 10 and Line Probe Assay. MIB-1 immunostaining was performed, and positive staining was defined as a cluster of two or more stained nuclei in the upper two thirds of the epithelial thickness. After verification of the diagnosis using the consensus histologic review and HPV detection as an objective confirmatory test, $31 \%$ of cases originally diagnosed as VIN1/VAIN1 were identified as being overdiagnosed. The sensitivity and the specificity of MIB-1 staining for identifying VIN1/VAIN1 were 0.96 and 0.90 , respectively. Seventy percent of VIN1 cases were associated with low-risk viral types. In contrast, the majority ( $84 \%)$ of VAIN1 cases were associated with high-risk HPVs. In conclusion,

Copyright (C) 2003 by The United States and Canadian Academy of Pathology, Inc.

VOL. 16, NO. 8, P. 735, 2003 Printed in the U.S.A

Date of acceptance: May 1, 2003.

Address reprint requests to: Edyta C. Pirog, M.D., Department of Pathology, New York Presbyterian Hospital-Weill Medical College of Cornell University, 525 E. 68th Street, New York, NY 10021; fax: 212-746-8359; e-mail: ecpirog@mail.med.cornell.edu.

DOI: 10.1097/01.MP.0000081051.55284.2A
MIB-1 staining is sensitive and specific for identifying VIN1/VAIN1, helpful in verifying the diagnosis in equivocal cases.

KEY WORDS: HPV, Low-grade vulvar intraepithelial neoplasia, Low-grade vaginal intraepithelial neoplasia, Ki-67, MIB-1, VAIN1, VIN1.

Mod Pathol 2003;16(8):735-741

Human papillomavirus (HPV) infection of vulvar skin and mucosa may clinically present either as exophytic condyloma (CON-A) or as a flat lesion currently termed vulvar intraepithelial neoplasia (VIN). The International Society for the Study of Vulvar Disease (ISSVD) (1) and the World Health Organization (2) endorsed the VIN terminology for noninvasive neoplastic vulvar lesions with subdivisions into VIN1 (mild dysplasia), VIN2 (moderate dysplasia), and VIN3 (severe dysplasia) depending on the level of involvement of the epithelium by the immature dysplastic cells. The ISSVD classification helped to standardize the terminology between different laboratories and eliminated numerous descriptive clinical diagnoses such as Bowen's disease, bowenoid papulosis, erythroplasia of Queyrat, and dystrophy with atypia, among others. Even though VIN terminology is useful and has been quickly adopted, there are still certain diagnostic problems. First, it is sometimes difficult to distinguish VIN2 from VIN3. In many cases the distinction is so subjective that some laboratories choose to combine the two grades and render the diagnosis as "high-grade vulvar intraepithelial neoplasia (VIN2-3)" or "high-grade squamous intraepithelial lesion (VIN2-3)" in parallel to the diagnostic scheme used for cervical lesions. Second, diagnosis of VIN1 has poor interobserver and intraobserver reproducibility. The diagnostic difficulty arises 
from subtle features of HPV cytopathic effect (koilocytosis) in the vulvar skin/mucosa, and lesser degree of nuclear atypia, as compared with the case of cervical low-grade squamous intraepithelial lesions (LSIL). In cases where nuclear atypia is minimal, the main differential diagnosis of VIN1 is "normal" skin/mucosa. In addition, presence of pseudokoilocytes in benign skin/mucosa may cause overdiagnosis. These diagnostic problems are clearly illustrated by a study of van Beurden et al. (3), in which $44 \%$ of vulvar biopsies originally diagnosed as "normal" received a diagnosis of VIN1 upon review, and $40 \%$ of cases originally diagnosed as VIN1 were reclassified as "normal." In another study (4), an audit of cases originally diagnosed as VIN1 was performed by the dermatopathologist and the surgical pathologists. In only 2 of 21 cases $(9.5 \%)$ was the original diagnosis validated; the diagnosis in the remaining cases was revised to squamous cell hyperplasia $(28 \%)$, psoriasis $(24 \%)$, lichen planus (14\%), and vesicular/bullous diseases (14\%).

The pathobiology of VIN1 has not been well studied, and association with specific HPV genotypes is not known. In contrast, other vulvar lesions have been extensively investigated. Vulvar condyloma acuminatum is a benign growth that has been shown to harbor low-risk HPVs, most commonly HPV6 or HPV $11(5,6)$. VIN3, on the other hand, is a premalignant lesion associated with high-oncogenic risk HPVs, specifically HPV $16(3,7,8)$. It is not clear, however, whether VIN1 is biologically related to benign condyloma, or if it is a precursor of VIN2-3.

Vaginal lesions associated with HPV infection are similarly challenging, and both underdiagnosis or overdiagnosis may result from subtle features of koilocytic atypia or presence of pseudokoilocytes, respectively. In a study by Nuovo et al. (9), lowgrade vaginal lesions were tested for HPV DNA by PCR, and only $63 \%$ of cases were found to be HPV positive, suggesting overdiagnosis in the remaining cases. The investigators observed that perinuclear haloes and mild nuclear atypia were not predictive of HPV DNA detection.

The purpose of this study was to evaluate the utility of MIB-1 immunostaining as an adjunct test to increase the diagnostic accuracy in equivocal cases of low-grade vulvar as well as vaginal intraepithelial neoplasia (VIN1/VAIN1). Next, we wanted to determine the distribution of low- and highoncogenic risk HPVs in VIN1/VAIN1 to acquire better understanding of the pathogenesis of these lesions. Finally, we were interested in identifying any subgroups within VIN1/VAIN1 cases based on either histologic or virologic characteristics. In particular, we wanted to determine whether dividing low-grade vaginal/vulvar intraepithelial neoplasia into two separate categories of "mild dysplasia" and "flat condyloma" has any biological significance.

Because any study of VIN1/VAIN1 potentially may be flawed by inclusion of non-neoplastic cases because of subjectivity of histologic diagnosis, our first objective was to identify the gold standard VIN1/VAIN1 and benign cases. The archival cases of VIN/VAIN and benign cases, including squamous cell hyperplasia and lichenoid lesions, were reviewed, and a consensus histologic diagnosis was obtained. The consensus diagnosis was further confirmed with HPV testing. In such validated gold standard cases, we determined the sensitivity and specificity of MIB-1 immunostaining for verifying the diagnosis. Finally, we performed genotyping of HPV DNA detected in VIN1/VAIN1 lesions using VIN3 cases as a positive control.

\section{MATERIALS AND METHODS}

\section{Case Selection}

The surgical pathology files of the Departments of Pathology at the Weill Medical College of Cornell University and Washington University School of Medicine were searched from 1997 to 2000 to identify successive vulvar/vaginal biopsies with the diagnosis of either benign or low-grade vulvar and vaginal intraepithelial neoplasia (VIN1 and VAIN1) and high-grade vulvar intraepithelial neoplasia (VIN3). The diagnoses were rendered by numerous faculty members with varying years of diagnostic experience. The study group consisted of 43 cases of combined VIN1 $(n=23)$ and VAIN1 $(n=20)$. The negative control group consisted of 20 benign cases, including squamous cell hyperplasia $(n=9)$; lichen planus $(n=4)$; and benign squamous mucosa $(n=7)$. The positive control group consisted of 6 cases of VIN3.

\section{Verification of Histologic Diagnosis}

To verify the histologic diagnoses, all cases were reviewed by two pathologists (SL and ECP) using standard criteria (10) to obtain a consensus diagnosis that was further correlated with the results of HPV testing. The study included only VIN/VAIN cases in which the consensus diagnosis was confirmed by positive HPV result (considered goldstandard positive cases) and benign cases in which the consensus diagnosis was confirmed by negative HPV result (considered as gold-standard negative cases). Cases of differentiated VIN were not included in the study.

\section{MIB-1 Immunohistochemistry}

MIB-1 immunohistochemical staining was performed on $4-\mu \mathrm{m}$ sections of formalin-fixed, 
paraffin-embedded specimens. The sections were subjected to heat-induced antigen retrieval and incubated in an automated stainer with MIB-1 antibody (Zymed Laboratories, Inc., San Francisco, CA) at a dilution of 1:300, stained with diaminobenzidine chromogen, and counterstained with hematoxylin. Presence of parabasal epithelial staining was used as a positive control. A presence of a cluster of two or more stained nuclei in the upper two thirds of the epithelial thickness was interpreted as a positive result (5).

\section{DNA Preparation}

Genomic DNA was prepared from two to three $4-\mu \mathrm{m}$ sections from each case using standard methods (5). Briefly, the slides were deparaffinized and stained with hematoxylin, and tissue was scraped with a sterile needle. The samples were incubated with $250-\mu \mathrm{L}$ proteinase $\mathrm{K}(1 \mathrm{mg} / \mathrm{mL})$ in $50 \mathrm{~mm}$ Tris-HCl, pH 8.0, 1 mm EDTA, and 0.5\% Tween 20 for 18 hours at $56^{\circ} \mathrm{C}$. After heat inactivation at $95^{\circ} \mathrm{C}$ for 10 minutes, $10 \mu \mathrm{L}$ of the supernatant was used for PCR. The entire tissue preparation was carried out in a specially dedicated laboratory to avoid PCR product carryover. Adequate DNA quality was established by PCR amplification of $\beta$-globin gene, resulting in a 96 -base pair product (11).

\section{HPV DNA Amplification}

Broad-spectrum HPV DNA amplification was performed using the Short PCR Fragment (SPF 10) primer set, as described previously (12). SPF 10 PCR amplifies a 65-base pair fragment from the L1 region of the HPV genome. Briefly, HPV DNA amplification was performed in a total volume of $50 \mu \mathrm{L}$ containing $10 \mu \mathrm{L}$ of isolated DNA, $10 \mathrm{~mm}$ Tris- $\mathrm{HCl}$ (pH 9.0), $50 \mathrm{~mm} \mathrm{KCl,} 2.0 \mathrm{~mm} \mathrm{MgCl}_{2}, 0.1 \%$ Triton $\mathrm{X}-100,0.01 \%$ gelatin, $200 \mu \mathrm{M}$ of each deoxynucleoside triphosphate, $15 \mathrm{pmol}$ of each of the forward and reverse primers (sequence as in Kleter et al. [12]), and 1.5 U of AmpliTaq Gold (Perkin Elmer). AmpliTaq Gold was activated by incubation at $94^{\circ} \mathrm{C}$ for 9 minutes. HPV DNA was amplified in 40 cycles of 30 seconds at $94^{\circ} \mathrm{C}, 45$ seconds at $52^{\circ} \mathrm{C}$, and 45 seconds at $72^{\circ} \mathrm{C}$, and a final extension of 5 minutes at $72^{\circ} \mathrm{C}$. Each experiment was performed with separate positive (plasmid HPV DNA) and negative $\left(\mathrm{H}_{2} \mathrm{O}\right)$ controls. PCR products were analyzed using $3 \%$ agarose gel electrophoresis. All HPV-negative cases were confirmed by the second PCR assay using standard DNA concentration as well as $10 \times$ diluted DNA sample to exclude presence of PCR inhibitors.

\section{HPV DNA Genotyping}

Samples identified as positive for HPV DNA were genotyped with the HPV-Line Probe Assay (Innoge- netics Inc., Alpharetta, GA). Twenty-five individual HPV genotypes (high-risk HPV: 16, 18, 31, 33, 35, 39, 45, 51, 52, 56, 58, 59, 66, 68, and 70; low-risk HPV: 6 , $11,34,40,42-44,53,54$, and 74) can be identified simultaneously in a single assay. The exact assay conditions were described previously (13). Briefly, $10 \mu \mathrm{L}$ of denatured HPV PCR product was hybridized $\left(60 \mathrm{~min}\right.$ at $50^{\circ} \mathrm{C}$ ) to genotype-specific probes immobilized as parallel lines on a nitrocellulose strip. After the washing step, the products of hybridization were visualized in a color reaction using alkaline phosphatase-streptavidin conjugate, 5-bromo-4-chloro-3-indolylphosphate, and nitroblue tetrazolium, which results in a purple precipitate. The results were assessed by aligning the strips with the standard grid (Fig. 1).

\section{RESULTS}

\section{Clinical Data}

The average age of patients with the diagnosis of VIN1 and VAIN1 was 45.1 years (range, 18-91) and 43.7 years (range, 18-81), respectively. History of intraepithelial neoplasia (either vulvar or cervical) was present in $25 \%$ of VIN1 and $19 \%$ of VAIN1 cases. A concurrent high-grade vulvar/vaginal intraepithelial neoplasia was identified in $28 \%$ of

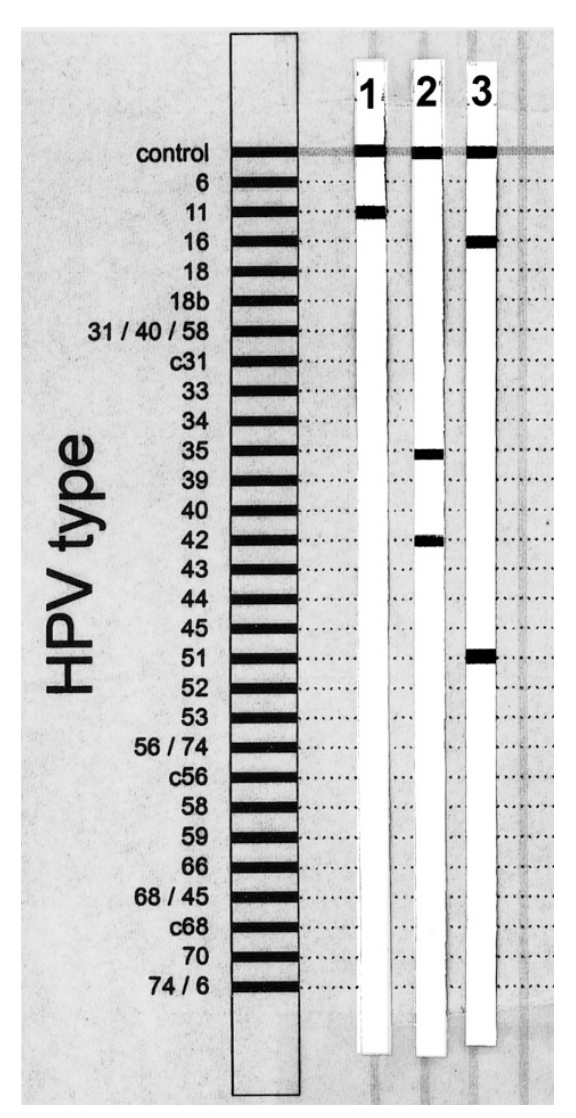

FIGURE 1. HPV genotyping using Line Probe Assay. Lane 1, HPV11; Lane 2, HPV35 and HPV42; Lane 3, HPV16 and HPV51. 
VIN1 and $10 \%$ of VAIN1 cases. The average age of the patients with VIN3 was 43.3 years (range, 2765). History of vulvar or cervical intraepithelial neoplasia was present in all cases. The average age of the patients with benign diagnosis was 61 years (range, 46-91). History of vulvar or cervical intraepithelial neoplasia was not recorded in any of the cases, and concurrent VIN3 was present in one case.

\section{Identification of Gold-Standard VIN1/VAIN1 Cases}

Thirty of 43 cases $(69 \%)$ originally diagnosed as VIN1/VAIN1 were confirmed as VIN1/VAIN1, and the remaining $13(31 \%)$ were identified as overdiagnosis and reclassified as benign based on the result of consensus review and negative HPV testing (Table 1). The reclassified cases included 11 originally diagnosed as VIN1 and 2 originally diagnosed as VAIN1. The revised diagnoses for these cases included squamous cell hyperplasia (1 case), lichen planus (2 cases), and benign squamous mucosa with reactive changes (10 cases). All cases originally diagnosed as VIN3 or benign were validated as such.

\section{MIB-1 Immunostaining in Gold-Standard VIN1/ VAIN1, VIN3, and Benign Cases}

MIB-1 positivity was identified in 29 of 30 goldstandard VIN1/VAIN1 cases (Table 1), 6 of 6 VIN3 cases, and 2 of 33 gold-standard benign cases (Fig. 2). The two MIB-1-positive benign cases had a diagnosis of squamous cell hyperplasia. The sensitivity and the specificity of MIB-1 positivity for identifying VIN1/VAIN1 lesions was 0.96 and 0.90 , respectively. There was no difference in MIB-1 staining pattern between cases positive for low-risk and high-risk HPVs.

HPV DNA Genotyping in Gold-Standard VIN1, VAIN1, and VIN3 Cases

The results of HPV genotyping are presented in Table 2 and Figure 1. Seventy percent of VIN1 cases

TABLE 1. Correlation of the Original Histologic Diagnoses with Gold Standard Diagnoses and Results of MIB-1 Immunostaining

\begin{tabular}{lccc}
\hline $\begin{array}{c}\text { Original } \\
\text { Diagnosis }\end{array}$ & $n$ & $\begin{array}{c}\text { Gold } \\
\text { Standard } \\
\text { Diagnosis* }\end{array}$ & $\begin{array}{c}\text { MIB-1 } \\
\text { Positive** }^{* *}\end{array}$ \\
\hline VIN1/VAIN1 & 30 & VIN1/VAIN1 & 29 \\
VIN1/VAIN1 & 13 & BENIGN & 0 \\
BENIGN & 20 & BENIGN & 2 \\
VIN3 & 6 & VIN3 & 6 \\
\hline
\end{tabular}

* Gold standard diagnosis was based on the results of consensus review and HPV testing.

** Presence of two or more stained nuclei in the upper two-thirds of the epithelial thickness was interpreted as a positive result. were associated with low-risk HPVs, and $84 \%$ of VAIN1 cases were associated with high-risk HPVs. All 6 cases of VIN3 were positive for high-risk HPVs. Mixed HPV type infection was identified in $20 \%$ of VIN1/VAIN1 and $30 \%$ of VIN3 cases, respectively.

\section{"Flat Condyloma" Versus "Mild Dysplasia"}

All gold-standard VIN1/VAIN1 cases were subclassified-using a consensus review-into "flat condyloma" (a lesion with koilocytic atypia but no evidence of dysplasia; $n=15$ ) or mild dysplasia (a lesion with koilocytic atypia in the superficial layers of the epithelium, loss of maturation with associated nuclear hyperchromasia, pleomorphism, and cellular crowding confined to the lower one third of the epithelium; $n=15$ ). The average age of the patients in both groups was 45 years. The frequency of past history of HPV infection was $40 \%$ in the flat condyloma group versus $53 \%$ in the mild dysplasia group $\left(P>.05\right.$ by $\left.\chi^{2}\right)$. The ratio of low-risk to high-risk HPVs was 1:2.7 in the flat condyloma group and 1:2.2 in the mild dysplasia group $(P>.05$ by $\chi^{2}$ ). The MIB-1 staining pattern in both groups was identical.

\section{DISCUSSION}

The histologic criteria to diagnose VIN1 are elaborated in the ISSVD classification of vulvar disorders, but the application of these criteria has considerable interobserver variability, and the diagnosis of VIN1 and VAIN1 continues to be a challenge when based on histopathologic criteria alone. In this study we have audited cases with the original diagnosis of VIN1/VAIN1 using a consensus histologic review and HPV DNA detection as an objective confirmatory test. We found that $31 \%$ of cases were overdiagnosed as VIN1/VAIN1. The medical and social ramifications of this finding are of great importance. Because VIN1/VAIN1 is related to the sexual transmission of a potentially oncogenic virus, women for whom the diagnosis is rendered face significant therapeutic, sexual, and social consequences. Although verification of the accuracy of the diagnosis could be performed with demonstration of HPV DNA by PCR, this technique requires special expertise and may not be always available. MIB-1 positivity has been extensively used as a surrogate marker of dysplasia in cervical biopsies. Notably, it has been used to differentiate pseudokoilocytic changes and atrophy from dysplasia $(14,15)$ and has been used to evaluate cauterized margins in cervical cone biopsies (16). In this study we evaluated the utility of the stain as an adjunct test for accurate diagnosis of VIN1/VAIN1.

MIB-1 is a monoclonal antibody recognizing Ki-67 nuclear nonhistone protein expressed 


\begin{tabular}{|c|c|c|c|}
\hline $\begin{array}{c}\text { Gold } \\
\text { Standard } \\
\text { Diagnosis }\end{array}$ & $n$ & $\begin{array}{c}\text { Low:High Risk } \\
\text { HPV Ratio* }\end{array}$ & HPV Genotypes \\
\hline VIN1 & 11 & $70: 30$ & $\begin{array}{l}\text { Low risk: } \\
\text { HPV6 }(\mathrm{n}=4) \text { HPV11 }(\mathrm{n}=1) \text { HPV44 }(\mathrm{n}=1) \\
\text { HPV74 }(\mathrm{n}=1) \\
\text { High risk: } \\
\text { HPV31 }(\mathrm{n}=1) \text { HPV68 }(\mathrm{n}=1) \\
\text { Mixed high/low risk types: } \\
\text { HPV16 + HPV51 }(\mathrm{n}=1) \\
\text { HPV unknown type }(\mathrm{n}=1)\end{array}$ \\
\hline VAIN1 & 19 & $16: 84$ & $\begin{array}{l}\text { Low risk: } \\
\text { HPV42 }(\mathrm{n}=1) \text { HPV44 }(\mathrm{n}=1) \text { HPV53 }(\mathrm{n}=1) \\
\text { High risk: } \\
\text { HPV16 }(\mathrm{n}=2) \text { HPV56 }(\mathrm{n}=2) \text {, HPV18 }(\mathrm{n}=3) \text {, } \\
\text { HPV31,59,68 }(\mathrm{n}=1 \text { each) } \\
\text { Mixed high/low and high/high risk types: } \\
\text { HPV16 + 66, HPV16 }+53+68, \text { HPV35 }+42 \text {, } \\
\text { HPV } 42+56 \text {, HPV56 }+66(\mathrm{n}=1 \text { each }) \\
\text { HPV unknown type }(\mathrm{n}=1)\end{array}$ \\
\hline VIN3 & 6 & $0: 100$ & $\begin{array}{l}\text { High risk: } \\
\text { HPV16 }(\mathrm{n}=3) \text {, HPV58 }(\mathrm{n}=1) \\
\text { Mixed high/low risk types: } \\
\quad \text { HPV16 }+6 \text {, HPV18 }+52+56+74(\mathrm{n}=1 \text { each })\end{array}$ \\
\hline
\end{tabular}

* Cases of mixed high/low risk HPV infection were counted as high risk cases.

throughout the mitotic cycle, with the exception of the $\mathrm{G}_{0}$ phase. Immunostaining with MIB-1 or polyclonal anti-Ki-67 antibodies yields the same results (17). In normal vulvar skin and vulvar/cervical mucosa, MIB-1 positivity is found exclusively in the parabasal nuclei of the squamous epithelium $(3,5$, 18-22). HPV infection results in increased proliferative activity of the squamous cells and extension of MIB-1-positive cells to the upper layers of the epithelium $(5,21,22)$. Presence of MIB-1-positive cells above the parabasal epithelial layer has been described in both VIN1 (3), and VIN2-3 (3, 18, 20, 23); however, these results were not correlated with HPV testing. Previous studies of vulvar condylomata and LSIL of the cervix showed a complete correlation between detection of HPV DNA and MIB-1 positivity that was defined as presence of MIB-1-positive cells in the upper two thirds of the epithelial thickness $(5,14)$. In the current study, we used the same definition of MIB-1 positivity and found an excellent correlation between HPV detection and MIB-1 positivity. The sensitivity of MIB-1 immunostaining for identification of VIN1/VAIN1 was 0.96 .

The differential diagnosis of VIN1 includes normal skin/mucosa and, among the most common disorders, squamous cell hyperplasia, lichen planus, psoriasis, and differentiated VIN. We detected positive MIB-1 staining in the upper two thirds of the epithelium in 2 of 9 cases of squamous cell hyperplasia. None of 4 cases of lichen planus were positive for MIB-1 in our study. The specificity of MIB-1 for detection of VIN1/VAIN1 was 0.90 . In addition, previous reports described only basal/parabasal MIB-1 stain- ing in psoriasis $(19,24)$ and differentiated VIN (23). These results indicate that MIB-1 is a specific marker of vulvar/vaginal dysplasia; however, in cases where the differential diagnosis includes squamous cell hyperplasia, HPV testing may be preferable for validation of the diagnosis.

Even though interpretation of MIB-1 positivity is highly reproducible, regardless of the pathologist experience, there are few instances that may result in false-positive interpretation of the staining. Tangential sectioning through the stromal papillae may result in apparent presence of positive nuclei in superficial layers of the epithelium. Examination of the overall tissue architecture may help to evade this potential interpretative error. In cases of vulvitis/vaginitis or lichenoid lesions, MIB-1-positive lymphocytes may be present throughout the epithelial thickness. High-power examination helps to identify these cells as nonepithelial.

The second goal of our study was to determine the distribution of low- and high-oncogenic risk HPVs in VIN1/VAIN1 to acquire insight into the pathobiology of these lesions. We found that VIN1 was most commonly associated with low-risk HPVs (70\%). This result suggests that flat VIN1 lesions are related to exophytic condylomata acuminata which are positive for low-risk HPVs in $77-88 \%$ of cases (5-7). For the same reason, it is unlikely that VIN1 is a precursor of VIN3, which is consistently associated with high-risk HPVs. Interestingly, $84 \%$ of VAIN1 lesions were found to be positive for highrisk HPVs, similar to cervical LSILs, which have been reported to be associated with high-risk HPVs in $83 \%$ of cases (25). 

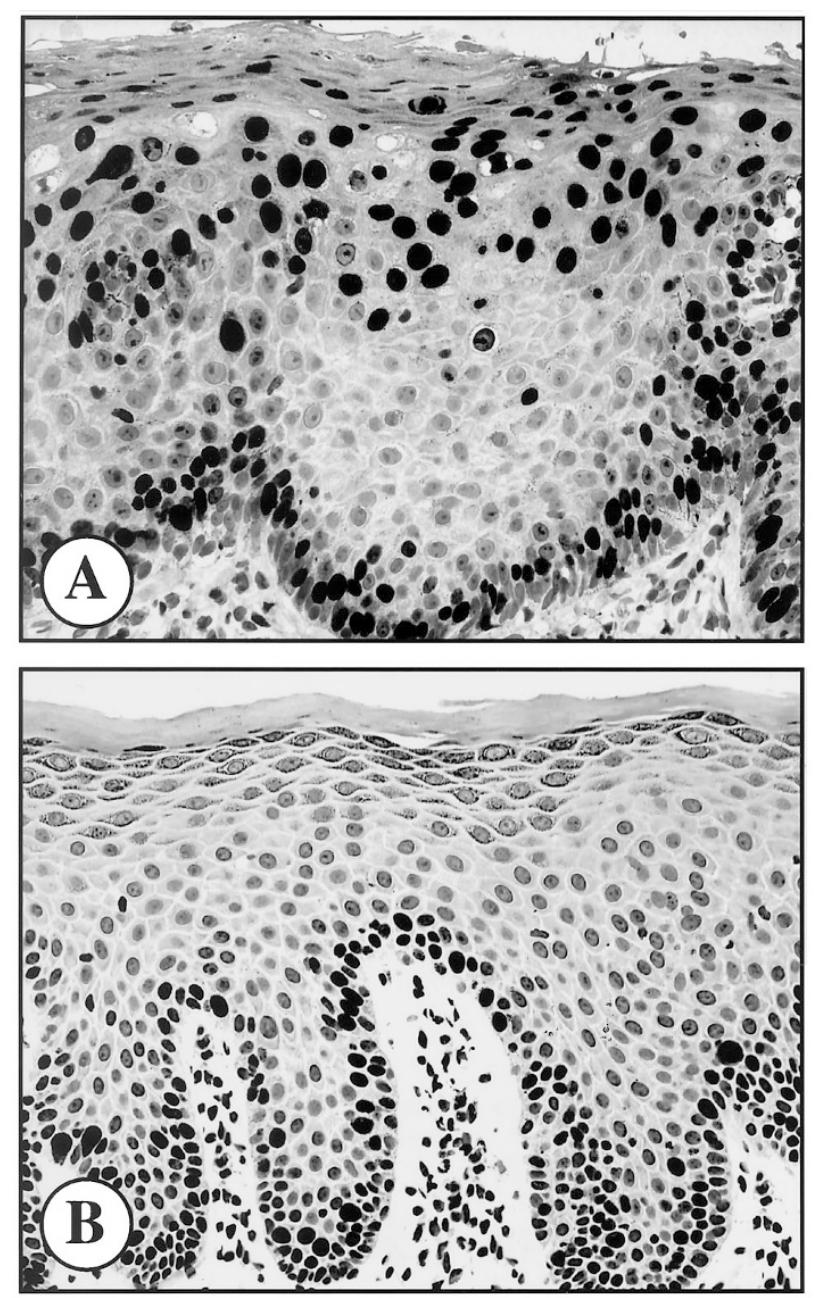

FIGURE 2. A, MIB-1 immunostaining of gold-standard VIN1. Positive staining is present in the parabasal layer as well as in the upper two thirds of the epithelial thickness (case categorized as MIB-1 positive). B, MIB-1 immunostaining of gold-standard benign skin (squamous cell hyperplasia). Positive staining is present only in the parabasal layer of the epithelium; in addition, few MIB-1-positive intraepithelial lymphocytes are present (case categorized as MIB-1 negative).

Finally, we were interested in identification of any subgroups within the VIN1/VAIN1 cases based on histologic, virologic, or clinicopathologic characteristics. The cases were substratified into flatcondyloma or mild-dysplasia categories. There was no difference in clinicopathological features of patients, HPV type distribution, or MIB-1-staining pattern between the two groups. Our results indicate that the distinction between "flat condyloma" and "mild dysplasia" may be an artificial one and has little biological significance.

In conclusion, results of previous studies and our current study indicate that even stringent morphological criteria fail to reliably predict the presence of HPV in vulvar/vaginal lesions $(3,5,9)$. Although the assessment of diagnostic accuracy could be performed with HPV detection by PCR, this technique has limited clinical use because of labor-intensive procedures and high cost. MIB-1 positivity corre- lates with the detection of HPV in both vulvar and vaginal low-grade squamous intraepithelial lesions with high sensitivity and specificity. Furthermore, MIB-1 immunostaining is routinely available, inexpensive, and reproducible. Our results clearly demonstrate that MIB-1 immunostaining is a beneficial adjunctive test that may be an important element of quality assurance in rendering diagnoses in equivocal cases.

\section{REFERENCES}

1. Wilkinson EJ. The presidential address. International Society for the Study of Vulvar Disease. J Reprod Med 1990;1989:35: 981-91.

2. Vulva. Epithelial tumors and related lesions. In: Scully RE, Bonfiglio TA, Kurman RJ, Silverberg SG, Wilkinson EJ, editors. Histological typing of female genital tract tumors. 2nd ed. New York: Springer-Verlag; 1994. p. 64-66.

3. van Beurden M, de Craen AJ, de Vet HC, Blaauwgeers JL, Drillenburg P, Gallee MP, et al. The contribution of MIB 1 in the accurate grading of vulvar intraepithelial neoplasia. J Clin Pathol 1999;52:820-4.

4. Micheletti L, Barbero M, Preti M, Zanotto Valentino MC, Chiringhello B, Pippione M, et al. Vulvar intraepithelial neoplasia of low grade: a challenging diagnosis. Eur J Gynaecol Oncol 1994;15:70-4.

5. Pirog EC, Chen YT, Isacson C. MIB-1 immunostaining is a beneficial adjunct test for accurate diagnosis of vulvar condyloma. Am J Surg Pathol 2000;24:1393-9.

6. McLachlin CM, Kozakewich H, Craighill M, O'Connell B, Crum CP. Histologic correlates of vulvar human papillomavirus infection in children and young adults. Am J Surg Pathol 1994;18:728-35.

7. Buscema J, Naghashfar Z, Sawada E, Daniel R, Woodruff JD, Shah K. The predominance of human papillomavirus type 16 in vulvar neoplasia. Obstet Gynecol 1988;71:601-6.

8. Hording U, Daugaard S, Junge J, Lundvall F. Human papillomaviruses and multifocal genital neoplasia. Int J Gynecol Pathol 1996;15:230-4.

9. Nuovo GJ, Blanco JS, Silverstein SJ, Crum CP. Histologic correlates of papillomavirus infection of the vagina. Obstet Gynecol 1988;72:770-4.

10. Wilkinson EJ. Premalignant and malignant tumors of the vulva. In: Kurman RJ, editor. Blaustein's pathology of the female genital tract. 6th ed. New York: Springer-Verlag; 1994. p. 87-130.

11. Bauer HM, Ting Y, Greer CE, Chambers JC, Tashiro CJ, Chimera J, et al. Genital human papillomavirus infection in female university students as determined by a PCR-based method. JAMA 1991;265:472-7.

12. Kleter B, van Doorn LJ, ter Schegget J, Schrauwen L, van Krimpen K, Burger M, et al. Novel short-fragment PCR assay for highly sensitive broad-spectrum detection of anogenital human papillomaviruses. Am J Pathol 1998;153:1731-9.

13. Kleter B, van Doorn LJ, Schrauwen L, Molijn A, Sastrowijoto $\mathrm{S}$, ter Schegget J, et al. Development and clinical evaluation of a highly sensitive PCR-reverse hybridization line probe assay for detection and identification of anogenital human papillomavirus. J Clin Microbiol 1999;37:2508-17.

14. Pirog EC, Baergen RN, Soslow RA, Tam D, DeMattia AE, Chen YT, et al. Diagnostic accuracy of cervical low grade squamous intraepithelial lesions is improved with MIB-1 immunostaining. Am J Surg Pathol 2001;26:70-5.

15. Mittal K, Mesia A, Demopoulos RI. MIB-1 expression is useful in distinguishing dysplasia from atrophy in elderly women. Int J Gynecol Pathol 1999;18:122-4. 
16. Mittal K. Utility of MIB-1 in evaluating cauterized cervical cone biopsy margins. Int J Gynecol Pathol 1999;18:211-4.

17. Rose DS, Maddox PH, Brown DC. Which proliferation markers for routine immunohistology? A comparison of five antibodies. J Clin Pathol 1994;47:1010-4.

18. van Hoeven KH, Kovatich AJ. Immunohistochemical staining for proliferating cell nuclear antigen, BCL2, and MIB-1 in vulvar tissues. Int J Gynecol Pathol 1996;15:10-6.

19. Scurry J, Beshay V, Cohen C, Allen D. Ki67 expression in lichen sclerosus of vulva in patients with and without associated squamous cell carcinoma. Histopathology 1998;32: 399-404.

20. Modesitt SC, Groben PA, Walton LA, Fowler WC Jr, Van Le L. Expression of MIB-1 in vulvar carcinoma and vulvar intraepithelial neoplasia III: correlation with clinical prognostic factors. Gynecol Oncol 2000;76:51-5.

21. Isacson C, Kessis TD, Hedrick L, Cho K. Both cell proliferation and apoptosis increase with lesion grade in cervical neoplasia but do not correlate with human papillomavirus type. Cancer Res 1996;56:669-74.

22. Mittal K, Palazzo J. Cervical condylomas show higher proliferation than do inflamed or metaplastic cervical squamous epithelium. Mod Pathol 1998;11:780-3.

23. Al-Ghamdi A, Miller D, Benedet JL, Clement PB, Gilks CB. Invasive vulvar squamous cell carcinoma in young women: a clinicopathological study of 21 cases [abstract]. Mod Pathol 2000;13(1).

24. Soini Y, Kamel D, Paakko P, Lehto VP, Oikarinen A, Vahakangas KV. Aberrant accumulation of p53 associates with Ki67 and mitotic count in benign skin lesions. Br J Dermatol 1994;131:514-20.

25. Solomon D, Schiffman M, Tarone R. Comparison of three management strategies for patients with atypical squamous cells of undetermined significance: baseline results from a randomized trial. J Natl Cancer Inst 2001;93:293-9.

\section{Book Review}

\section{Pasqualini JR, editor: Breast Cancer: Progno- sis, Treatment, and Prevention, $656 \mathrm{pp}$, New York, Marcel Dekker, Inc., 2002 (\$165.00).}

Pasqualini and colleagues have assembled a significantly unique collection of reviews in breast cancer. In 17 widely ranging chapters, only occasionally overlapping, the authors provide indepth reviews of the many-faceted hormonal and molecular influences on breast tissue, both normal and malignant.

The authors intend their book to target oncologists, gynecologists, general clinicians, biologists, physiologists, and other advanced students. The majority of the chapters are relatively sophisticated reviews in basic science. Paradoxically, this is why the book may be most useful to clinicians whose available standard texts on breast cancer tend to emphasize reviews of clinical trials. Contrastingly, this text should gain readership from clinicians, particularly those working on the cusp of new patient-focused research, translating new molecular information into patient clinical trials.
The chapter on "BRCA-1, BRCA-2 and Hereditary Breast Cancer," by Bove et al., is particularly noteworthy; representing one of the most cogent and readable chapters on this issue the reviewer has had the good fortune to read. This chapter, as do many in this text, pushes the reader into regions of basic science that are new, and to fully understand may require some study beyond a cursory reading.

Each of the above-targeted specialists that the authors have identified can benefit from this book. But it would seem clinical researchers, particularly those doing translational research, will find this text most useful in their own endeavors. The book is recommended to those very clinical researchers and should be available in any academic library where such clinical researchers reside.

\author{
Ronald L. Stephens \\ Lawrence Memorial Oncology Center \\ Lawrence, Kansas \\ University of Kansas Medical Center \\ Kansas City, Kansas
}

